OPEN ACCESS

Edited by:

Eric Barrey,

INRA UMR 1313 Genetique Animale et Biologie Integrative, France

Reviewed by:

Javier Traba

Autonomous University of Madrid,

Spain

Arnon Henn,

Technion Israel Institute of Technology, Israel

*Correspondence:

Florence Apparailly

Florence.apparailly@inserm.fr

Maroun Khoury

mkhoury@uandes.c

Specialty section:

This article was submitted to

Mitochondrial Research,

a section of the journal

Frontiers in Physiology

Received: 08 July 2021 Accepted: 22 September 2021 Published: 03 November 2021

Citation:

Duroux-Richard I, Apparailly F and Khoury M (2021) Mitochondrial

MicroRNAs Contribute to Macrophage Immune Functions Including Differentiation, Polarization,

and Activation

Front. Physiol. 12:738140

doi: $10.3389 /$ fphys.2021.738140

\section{Mitochondrial MicroRNAs Contribute to Macrophage Immune Functions Including Differentiation, Polarization, and Activation}

\author{
Isabelle Duroux-Richard ${ }^{1}$, Florence Apparailly ${ }^{1,2 *}$ and Maroun Khoury ${ }^{3,4,5 *}$ \\ ${ }^{1}$ IRMB, INSERM, Université de Montpellier, CHU Montpellier, Montpellier, France, ${ }^{2}$ Clinical Department for Osteoarticular \\ Diseases, University Hospital of Montpellier, Montpellier, France, ${ }^{3}$ Laboratory of Nano-Regenerative Medicine, Faculty \\ of Medicine, Universidad de Los Andes, Santiago, Chile, ${ }^{4}$ Cells for Cells and REGENERO, The Chilean Consortium \\ for Regenerative Medicine, Santiago, Chile, ${ }^{5}$ MPACT, Center of Interventional Medicine for Precision and Advanced Cellular \\ Therapy, Santiago, Chile
}

A subset of microRNA (miRNA) has been shown to play an important role in mitochondrial (mt) functions and are named MitomiR. They are present within or associated with mitochondria. Most of the mitochondrial miRNAs originate from the nucleus, while a very limited number is encoded by mtDNA. Moreover, the miRNA machinery including the Dicer and Argonaute has also been detected within mitochondria. Recent, literature has established a close relationship between miRNAs and inflammation. Indeed, specific miRNA signatures are associated with macrophage differentiation, polarization and functions. Nevertheless, the regulation of macrophage inflammatory pathways governed specifically by MitomiR and their implication in immune-mediated inflammatory disorders remain poorly studied. Here, we propose a hypothesis in which MitomiR play a key role in triggering macrophage differentiation and modulating their downstream activation and immune functions. We sustain this proposition by bioinformatic data obtained from either the human monocytic THP1 cell line or the purified mitochondrial fraction of PMAinduced human macrophages. Interestingly, 22\% of the 754 assayed miRNAs were detected in the mitochondrial fraction and are either exclusively or highly enriched cellular miRNA. Furthermore, the in silico analysis performed in this study, identified a specific MitomiR signature associated with macrophage differentiation that was correlated with gene targets within the mitochondria genome or with mitochondrial pathways. Overall, our hypothesis and data suggest a previously unrecognized link between MitomiR and macrophage function and fate. We also suggest that the MitomiR-dependent control could be further enhanced through the transfer of mitochondria from donor to target cells, as a new strategy for MitomiR delivery.

Keywords: miRNA, mitochondria, macrophage, inflammmation, mitochondria transfer, microRNA, MitomiR 


\section{MITOCHONDRIA AS KEY ORGANELLES IN MACROPHAGE FUNCTIONS}

Mitochondria are crucial cellular organelles that act as metabolic hubs and signaling platforms. Beyond the fateful endosymbiosis theory (Gray et al., 1999; Roger et al., 2017), mitochondria are multitasking, highly dynamic organelles that continually adjust their morphology, function, number and even their cellular host, in response to metabolic needs and environmental changes (Caicedo et al., 2017). Hence, their functions go beyond cellular ATP production and energy metabolism. In fact, they have indispensable roles in the immune system, especially in regulating macrophage polarization and responses to infection, tissue damage and inflammation.

\section{MITOCHONDRIA TRIGGERING MACROPHAGE POLARIZATION}

During pro-inflammatory activation of macrophages (so-called M1 polarization), aerobic glycolysis is activated (Van den Bossche et al., 2016; Liu Y. et al., 2021). This metabolic switch involves increased glucose uptake and pyruvate conversion to lactic acid, providing macrophages with the sufficient energy necessary for their anti-bactericidal activity (He et al., 2021). Moreover, the M1-type activation of macrophages inhibits mitochondrial OXPHOS and promotes mitochondrial nitric oxide (NO) production, thus preventing repolarization toward the antiinflammatory M2 phenotype (Van den Bossche et al., 2016).

M2 macrophages have a complete tricarboxylic acid (TCA) cycle involving IL-4-mediated fatty acid oxidation (FAO) and mitochondrial energy metabolism as opposed to M1 macrophages (Jha et al., 2015). IL-4 induces ATP citrate lyase activation and oxidative phosphorylation (OXPHOS). In addition, it increases acetyl coenzyme A (acetyl-CoA) synthesis for histone acetylation, which is a key epigenetic regulator of the expression of several M2 marker genes. M2 macrophages maintain anti-inflammatory responses by obtaining most of their energy from FAO and oxidative metabolism (Qing et al., 2020).

IL-25 increases the mitochondrial respiratory capacity and oxygen consumption rate of macrophages and the production of NAD/NADH and ATP (Feng et al., 2017). This leads to the secretion of a large number of anti-inflammatory factors by macrophages for M2 polarization. Furthermore, the inhibition of NO production improves mitochondrial function and reprogramming into M2 macrophages (Mehla and Singh, 2019).

Mitophagy, a mechanism that selectively removes damaged mitochondria from cells, has also been related to macrophage polarization. During M1 polarization, mitophagy inhibition increases mitochondrial mass and decreases the expression of glycolysis-related genes and proinflammatory cytokines (Meng et al., 2021).

Although mitochondria are expected to act as a main target in macrophage regulation, the exact molecular mechanisms by which they regulate macrophage polarization is still not completely elucidated.

\section{MITOCHONDRIA ROLE IN THE INTERPLAY BETWEEN MACROPHAGE METABOLISM AND HOST DEFENSE MECHANISM}

Mitochondria are implicated in the tight interplay between host metabolic modifications and immune responses during bacterial infection (Ramond et al., 2019). Recently, it has been demonstrated that intracellular bacterial pathogens are able to modulate mitochondrial functions to maintain their replicative niche. Infection induces mitochondrial changes in infected macrophages, triggering modifications of the host metabolism that lead to important immunological reprogramming (Ramond et al., 2019). Indeed, stimulation by the bacterial toxin nigericin, or LPS, induced the NOD-like receptor family pyrin domaincontaining 3 (NLRP3) to interacts with mitochondria via the mitochondria-associated adaptor protein MAVS, leading to ASC (Apoptosis-associated speck like protein) polymerization and downstream activation of caspase 1 and cytokine production (Subramanian et al., 2013). Assembly of the NLRP3 complex leads to the autocatalytic activation of caspase- 1 and then the pro-inflammatory cytokines IL-1 $\beta$ and IL-18 (Kelley et al., 2019).

The uncoupling proteins (UCP), a subfamily of mitochondrial proteins, also plays a pivotal role in reprogramming macrophages during infection (Pecqueur et al., 2009). Upon LPS activation, macrophages downregulate UCP2 transcription, leading to a control of the mitochondria-derived reactive oxygen species (Emre and Nübel, 2010). Moreover, UCP2-(-/-) macrophages are more prompt to clear $S$. typhimurium intracellular infection (Arsenijevic et al., 2000).

\section{INVOLVEMENT OF MicroRNAs IN THE REGULATION OF MITOCHONDRIAL PROCESSES}

MicroRNA (miRNA) are evolutionarily conserved small non-coding RNAs, which regulate gene expression posttranscriptionally through mRNA degradation or translational inhibition (Bartel, 2004). Several studies demonstrate the presence of miRNAs in various organelles, including the nucleus, endoplasmic reticulum and the mitochondria. Mitochondriaassociated endoplasmic reticulum membranes (MAM) are sites of contact where the endoplasmic reticulum domains interact with the mitochondria, facilitating communication between these two organelles (Gao P. et al., 2020). Recently, these sites have shown to contain a substantial number of miRNAs, which may be re-distributed in response to cellular stress and metabolic demands (Wang et al., 2020).

There are three main types of miRNAs enriched in the mitochondrial fractions (MitomiR): (1) They can originate from the nuclear genome and mature in the cytoplasm to target cellular genes that modulate mitochondrial functions (nucMiR), (2) they can translocate into the mitochondria to target mitochondrial genes and functions (nuc-MitomiR), or (3) they can be transcribed from the mitochondrial genome and target 
mitochondrial genes and functions (mt-MitomiR) (Bandiera et al., 2011; Figure 1).

The subclass of MitomiRs that originate from the nuclear genome that mature and regulate the expression of genes associated with mitochondrial pathways in the cytoplasm compartment is the most abundant. Numerous studies report and demonstrate their involvement in mitochondrial functions and activity, such as in apoptosis. Mitochondria play an important role in apoptosis progression, through the mitochondrial fission/fusion dynamics and the release of pro-apoptotic factors (Sheridan and Martin, 2010). For example, miR-200a-3p, miR125b, miR-30 family, miR-485-5p, miR-195, and miR-140 regulate mitochondrial fission/fusion dynamics by targeting mitochondrial factors such as the mitochondrial fission factor (MFF) (Lee et al., 2017), the mitochondrial $18 \mathrm{kDa}$ protein (MTP18) (Duroux-Richard et al., 2016), the dynamin-1-like protein (DRP1) (Li et al., 2010), the mitofusin (MFN) 1 and 2 (Li et al., 2014; Zhao et al., 2017; Purohit et al., 2019). As the heart is the organ with a perpetual high energy requirement, mitochondria occupy a large portion of cardiomyocytes, and are located between the myofibrils and just below the sarcolemma (Gustafsson and Gottlieb, 2008). Hence, strategic positioning and abundance of mitochondria ensure a highly efficient localized ATP delivery system to support contraction, metabolism, and ion homeostasis (Andrienko et al., 2003). The involvement of miRNAs in the energy control of mitochondria is therefore crucial in those cells. Indeed, in cardiomyocytes, upon apoptotic stimulation, miR-140 negatively regulates MFN1 and controls the release of endonuclease $G$, which participates in causing DNA fragmentation, and leads to apoptosis (Li et al., 2014). Following the anti-tumor drug Doxorubicin (DOX) treatment, miR-532-3p directly targets the caspase recruitment domain (ARC) and participates in DOX-induced mitochondrial fission and apoptosis. MiR-499-5p attenuates mitochondrial fission and DOX cardiotoxicity through p21 targeting (Wan et al., 2019).

\section{MitomiR AND CELLULAR FUNCTIONS}

Apoptosis is also related to the pathogenesis of many diseases such as tumors, cardiovascular disorders and inflammatory responses, and miRNAs participate in the regulation of mitochondria-mediated apoptosis and metabolism. The miR30 family regulates apoptosis through the targeting of the mitochondrial fission machinery leading to a suppression in the expression of p53 and its downstream target DRP1 (Li et al., 2010).

In human monocytes upon inflammatory signal, miR125b attenuates mitochondrial respiration and promotes mitochondrial hyper-fusion through the silencing of both the BH3-only proapoptotic protein BIK and the pro-fission protein MTP18, leading to macrophage apoptosis (Duroux-Richard et al., 2016). In renal tubular cells, under hypoxia, MTP18 is silenced by miR-668, preserving mitochondria from fragmentation and

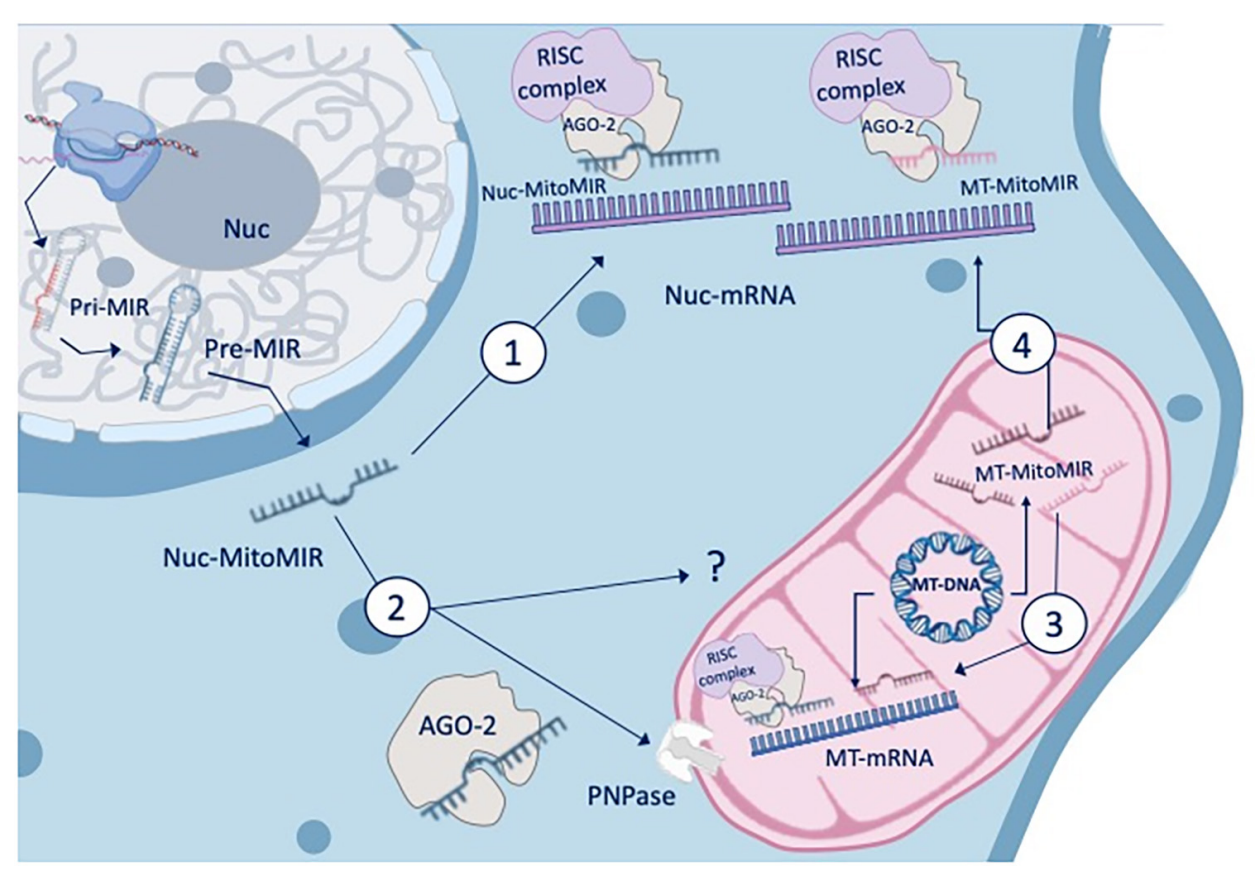

FIGURE 1 | MitomiR and gene regulation. The three main types of miRNAs that are enriched in the mitochondrial fractions can either originate from the nuclear genome and mature in the cytoplasm to target cellular genes that modulate mitochondrial functions (nuc-MiR) or translocate into the mitochondria to target mitochondrial genes and functions (nuc-MitomiR), or be transcribed from the mitochondrial genome and target mitochondrial genes and functions (MT-MitomiR). The AGO2 protein, an endonuclease, has been reported to colocalize with mitochondria, and to be associated with small mtRNA. RNA and MitomiR import into mitochondria is facilitated by multi-subunit RNA import complex (RIC), through a pathway independent of protein import and recently, through AGO2 and the protein PNPase (poly nucleotide phosphorylase). 
cell death (Wei et al., 2018). In addition, it has been shown that miR-338 targets cytochrome c oxidase IV, a key nuclear-encoded protein within the electron transfer chain in mitochondria, which is involved in ATP production (Aschrafi et al., 2008).

The first evidence that miRNAs can be imported into mitochondria and regulate mtRNA expression was demonstrated in the mtDNA-lacking $206 \rho^{\circ}$ cell line. Several nuc-MitomiR, such as miR-181c-5p and miR-146a-5p were identified in mitochondria and target mitochondrial RNAs and mitochondriaassociated mRNAs encoded by nuclear genes (Dasgupta et al., 2015). In 2014, Das et al. (2014) demonstrated that miR-181c encoded in the nucleus but matured in the cytosol, translocates into the mitochondria regulating the expression of mitochondrial genes such as $m t$-COX1 (known as mitochondrially encoded cytochrome c oxidase I). Using an in vivo systemic miR-181c delivery to the heart, enforced expression of miR-181c leads to an alteration in oxygen consumption, ROS production, matrix calcium and mitochondrial membrane potential of mitochondria isolated from cardiac cells (Das et al., 2014). More recently it has been shown that all members of the miR-181 family can alter the myocardial response to oxidative stress, by either targeting $m t$ COX1 (miR-181c) or tensin homolog gene PTEN (miR-181a and miR-181b) (Das et al., 2017).

\section{IDENTIFICATION OF MITOCHONDRIAL-ENRICHED MicroRNAs}

To identify mitochondrial-enriched miRNAs, miRNome studies of mitochondrial and cytosolic RNA fractions from the same cells were performed. nuc-MitomiR signatures, such as miR494, miR-1275 and miR-1974, were enriched in mitochondrial fractions (Bandiera et al., 2011). Subcellular trafficking and cellular dynamics of intracellular miRNA translocation depends on AGO2 levels and its status of phosphorylation. The AGO2 protein, an endonuclease shared across multiple species necessary for RNAi, has been reported to colocalize with mitochondria and to be associated with small mtRNA (Maniataki, 2005; Bandiera et al., 2011). In addition, it has been shown that RNA import into mitochondria is facilitated by multi-subunit RNA import complex (RIC), such as pathways independent of protein import (Mukherjee et al., 2007), co-localization of AGO2 and AGO3 (Bandiera et al., 2011), and recently, AGO2 and the protein PNPase (poly nucleotide phosphorylase) (Shepherd et al., 2017).

The functional RNAs encoded by the mitochondrial genome are mainly ribosomal RNAs (rRNAs) and transfer RNAs (tRNAs). The AGO2 protein has been shown to be associated with mitochondrial tRNAs in the cytoplasm. In addition, new types of RNA of mitochondrial origin have recently been identified. These are double-stranded RNAs encoded in mitochondria that are capable of triggering antiviral signaling in humans (Dhir et al., 2018). Interestingly, Bandiera et al. (2011) profiled the expression of miRNAs in mitochondrial fractions purified from HeLa cells and interrogated their genomics to better understand the molecular basis underlying their mitochondrial localization. They showed that miR-1974, miR-1977, and miR1978 have a perfect match in the mitochondrial genome with two mitochondrial tRNA genes, TRNE and TRNN, and with a stretch of the mitochondrial rRNA sequence RNR1 (Bandiera et al., 2011).

Thus, the post-transcriptional regulation of miRNAs directly into the mitochondria, or next to the mitochondria, especially in MAMs, allows the rapid expression of mitochondrial genome to be adjusted according to the metabolic conditions and demands of the cell. The importance of metabolism in deciding the fate of immune populations is very clear. Under conditions of stress, macrophages adjust their metabolism to meet the energy demand, necessary for their activation and functions.

\section{MitomiR PROFILING OF MITOCHONDRIA EXTRACTED FROM MONOCYTIC CELL LINE}

MiRNAs play pivotal roles in regulating macrophage functions, including differentiation, polarization, recruitment, and activation of inflammation (Self-Fordham et al., 2017). The ability of the mitochondria to modify cellular metabolic profile, thereby allowing an appropriate response, is crucial for the correct establishment of immune responses. However, little is known about mitomiR involvement when taking into account macrophage functions, such as differentiation. Thus, in this review, to provide novel insight into macrophage differentiationspecific mitomiR signatures and associated mitochondrial pathways, we performed a global miRNA profiling on extracted mitochondria and total cell content of human macrophages. The THP-1 monocytic cell line was treated with phorbol12-myristate-13-acetate (PMA) to induce differentiation into macrophages.

The mitochondrial fraction of the cells was enriched and purified using anti-TOM22 microbeads (Miltenyi Biotec). Briefly, $2 \times 10^{6}$ were required to allow purification of the total fraction while $40 \times 10^{6}$ cells were used for the isolation of the mitochondrial fraction. PMA-differentiated and undifferentiated THP-1 cells were centrifuged at $300 \mathrm{~g}$ at $4^{\circ} \mathrm{C}$ for $10 \mathrm{~min}$, after homogenization in ice-cold lysis-buffer supplemented with antiprotease cocktail using 26G and 30G needles and $1 \mathrm{ml}$ syringes (5 times round trips for each size). After checking that at least half of the effective lysis is achieved, the total cell fraction was obtained following centrifugation at $13,000 \mathrm{~g}$ for $5 \mathrm{~min}$ at $4^{\circ} \mathrm{C}$. The obtained pellets were resuspended in $700 \mu \mathrm{l}$ of Qiazol (Qiagen). In parallel, the mitochondrial cell fraction purification is carried out directly on the cell lysate by anti-TOM22 magnetic labeling (Miltenyi Biotec), according to the manufacturer's instructions. After mitochondrial fraction elution, samples were centrifugated at $13,000 \mathrm{~g}$ for $5 \mathrm{~min}$ at $4^{\circ} \mathrm{C}$, and mitochondria pellets resuspended in $700 \mu \mathrm{l}$ of Qiazol for RNA extraction, using the miRNeasy kit (Qiagen). We performed the global miRNA profiling by considering 754 human miRNAs on extracted mitochondria and compared with total cell content. Total 500 ng RNA per sample were reverse transcribed to cDNA with Megaplex 
Human Pool A and B stem loop RT primers and TaqMan MicroRNA RT kit (Life Technology), and miRNA expression profiles were analyzed using the $\operatorname{TaqMan}^{\circledR}$ Array Human MicroRNA Card Set v3.0, according to the manufacturer's instructions. ${ }^{1}$ To address differences, the distribution of miRNA expression was visualized using unsupervised hierarchical clustering (Figure 2A). Data showed that $78 \%$ of miRNAs were very poorly detected in the mitochondrial fraction. The supervised hierarchical clustering analysis showed that 22 miRNAs are increased or decreased during macrophage differentiation in the mitochondrial compartment (Figure 2A, miRNAs name in right top panel). A miRNA profiling between total cell content and purified endoplasmic reticulum was also performed and showed no enrichment of the identified MitomiR (data not show), suggesting that these miRNAs are specific for mitochondria. Using ingenuity pathway analysis (IPA) tool, ${ }^{2}$ we examined which biological pathways were affected with the twenty-two identified MitomiRs and constructed mRNA-miRNA networks and miRNA-target gene interactions (Figure 2B). This analysis highlighted 9 (miR-331-5p, miR-18a-8p, miR132, miR-29b-3p, miR-17-5p, miR-186-5p, miR-124-3p, miR192-5p, and miR-23a-3p) and 4 (miR-494-3p, miR-214-3p,

${ }^{1}$ https://www.ncbi.nlm.nih.gov/geo/query/acc.cgi?acc=GSE182255

${ }^{2} \mathrm{http}: / /$ www.ingenuity.com
miR-16-5p, and miR-154-5p) MitomiRs were down- and upexpressed upon macrophage differentiation, respectively, and were potentially linked to key genes associated with regulation, permeabilization, morphology, depolarization, development, quantity, and transmembrane potential of mitochondria.

\section{MitomiR AND MACROPHAGE FUNCTIONS}

Interestingly, among these thirteen MitomiRs, miR-494 is probably one of the most described nuc-MitomiR. It is enriched in mitochondrial fractions and controls cell differentiation and mitochondrial functions in different cell types. It regulates mitochondrial biogenesis by silencing mtTFA and Foxj3 during myocyte differentiation and skeletal muscle adaptation to physical exercise (Yamamoto et al., 2012). In ARPE-19 cells, a spontaneously arising retinal pigment epithelia (RPE) cell line, miR-494 is enriched in mitochondria and present in extracellular vesicles released by cells treated with rotenone to induce mitochondrial injury (Ahn et al., 2021). Thus, in addition to being the most frequently identified MitomiR (Geiger and Dalgaard, 2017), miR-494 is also involved in macrophage polarization and functions. Shao et al. (2020) showed that miR-494 enhances M1 macrophage polarization via NRDP1 targeting in an
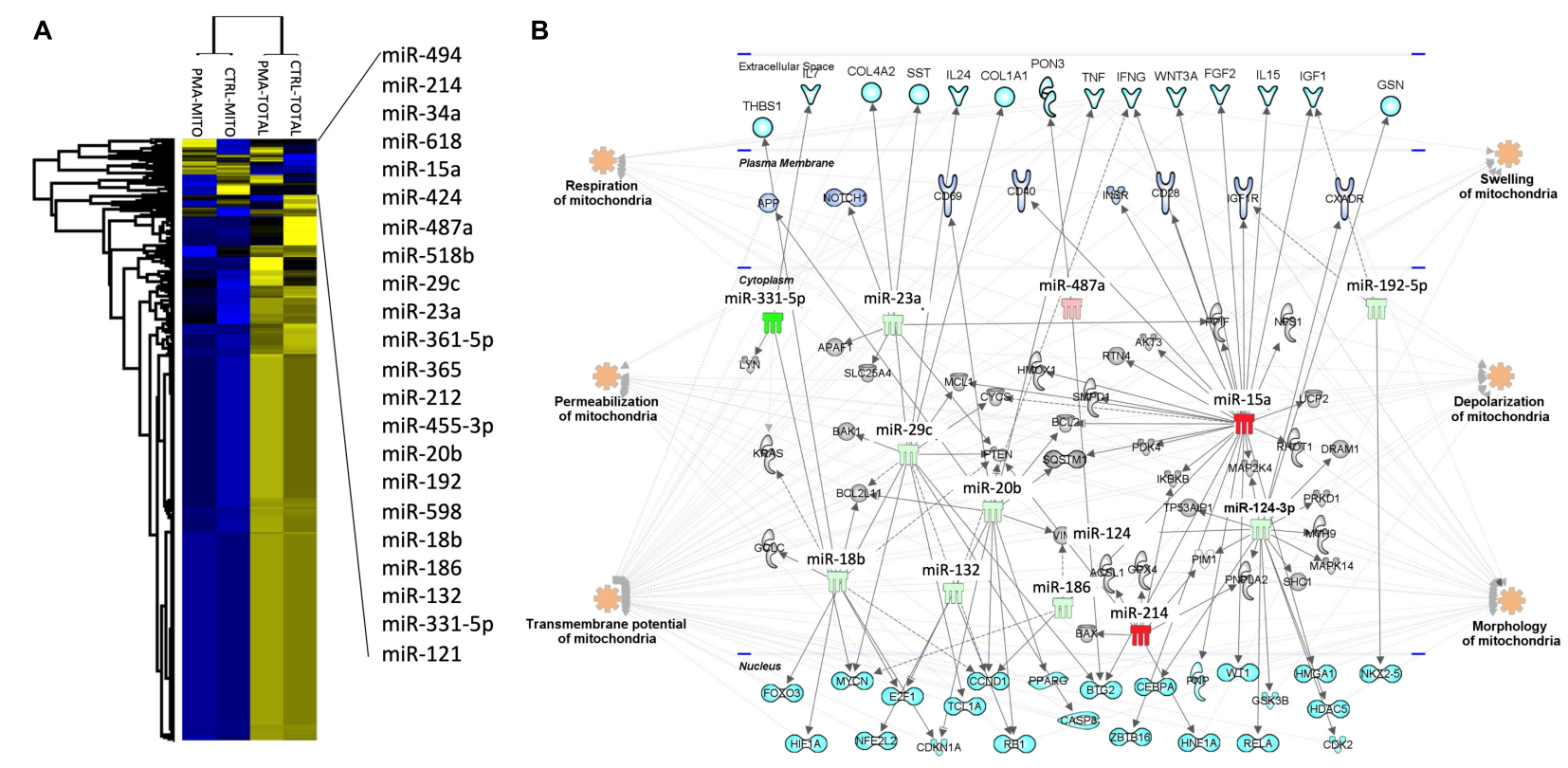

FIGURE 2 | Macrophage mitomiRs signature: The human monocytic cell line THP-1 cells was differentiated into THP-1 derived macrophages with 40 ng/mL phorbol 12-myristate 13-acetate (PMA) over-night in RPMl 1640 Medium GlutaMAX ${ }^{\mathrm{TM}}$ Supplement with $10 \%$ FBS at $37^{\circ} \mathrm{C}$ and $5 \%$ CO2. Total cellular and mitochondrial enrichment fractions were prepared at $4^{\circ} \mathrm{C}$ using a MACS Mitochondria kit (Miltenyi), and total RNA including miRNAs were extracted using miRNeasy kit (Qiagen). miRNA quantification was performed using the TaqMan ${ }^{\circledR}$ Array Human MicroRNA Card Set v3.0 (Applied Biosystems), according to the manufacturer's instructions. (A) Using Perseus software, a non-supervised hierarchical analysis of the distribution of total and mitochondria enrichment cell fractions based on relative log2 expression of 762 human miRNAs significantly modified by PMA compared to untreated controls. Yellow and blue represent the increase and decrease in expression, respectively. (B) miRNA-mRNA networks generated with Ingenuity Pathway Analysis (IPA) for the 22 macrophage-specific mitomiRs. IPA analysis was performed to investigate Mt-miRNA associated genes, filters were set to include experimentally observed or high-confidence-predicted miRNA-mRNA interaction partners, associated with mitochondria biological pathways. miRNAs were colored in red or green depending on whether they were upregulated or downregulated in mitochondria fractions following macrophage differentiation, respectively. 
intracerebral hemorrhage mice model. miR-494 also regulates inflammatory responses by targeting the phosphatase and PTEN and suppresses LPS-induced nuclear factor (NF)- $\kappa \mathrm{B}$ signaling, in LPS-induced inflammation in mouse macrophages (Zhang et al., 2019). Interestingly, PTEN expression is strongly regulated by several miRNAs, including miR-214, which we found enriched in the mitochondrial fraction too. Down-regulation of PTEN expression leads to LPS-induced AKT1 activation and inflammation (Fang et al., 2018). Furthermore, Lu et al. (2014) showed that the effect of macrophage polarization associated with the anti-cancer drug Norcantharidin on anti-hepatocellular carcinoma was due to increased miR-214 expression, which resulted in the inhibition of $\beta$-catenin signaling pathway. Recently, three studies demonstrated the key role of miR214, miR-487a, and miR-124 in M2 macrophage polarization. The long non-coding RNA NEAT1 promotes M2 macrophage polarization by sponging miR-214, which silences $B 7-H 3$ (alias CD276), thus accelerating multiple myeloma progression via the JAK2/STAT3 signaling pathway (Gao Y. et al., 2020). MiR$487 \mathrm{a}$ is enriched in M2 macrophage-derived exosomes, which promote gastric cancer proliferation and tumorigenesis (Yang et al., 2021). Finally, miR-124 contributes to M2-type polarization of monocytic cells in normal conditions and during allergic inflammation (Veremeyko et al., 2013).

To study the mitochondrial signaling pathways and to shed light on the implication of MitomiRs on the regulation of mitochondrial functions, we performed an in silico analysis with the two most described MitomiRs, miR-494 and miR-214, whose expressions are up- and down-expressed, respectively, during monocyte differentiation into macrophage. Using IPA, we constructed a $m t$-mRNA-miRNA networks and a miRNA-target $m$ t-gene interactions using putative miR-214 and miR-494 direct and indirect targets (Figure 3). Interestingly, miR-214-3p and miR-494-3p putatively target two $m t$-genes: the cytochrome $\mathrm{C}$ oxidase subunit I ( $m$ t-CO1, alias COX1) and subunit III (mt-CO3, COX3), respectively. Human cytochrome c oxidase (COX) is composed of 13 subunits, including three catalytic subunits I-III ( $m t-C O 1, m t-C O 2$, and $m t$-CO3) encoded by mitochondrial DNA and ten nuclear-coded subunits by nuclear DNA (Kadenbach and Hüttemann, 2015). Furthermore, COX phosphorylation is described as having a strong influence on mitochondrial respiration and controlling macrophage function during inflammatory stimuli.

For example, the osteopontin, a cell attachment protein, that trigger a number of signal transduction pathways controlling survival, proliferation and migration of macrophages (Gao et al., 2007), decreases its expression in the presence of $m t-C O 1$ and $m t-C O 3$ in RAW264, murine macrophages, through a CD44-dependent transcriptional regulatory mechanism of the mitochondrial $\mathrm{H}$ strand (Gao et al., 2003). In macrophages, endotoxin-stimulated NO production inhibits cellular respiration and mitochondrial electron transport by inhibiting cytochrome c oxidase activity, and inhibits $m t$-CO1 expression at the mRNA and protein levels, determining the host inflammatory response (Wei et al., 2002).

Finally, a RNA sequencing study of human macrophage subpopulation isolated from bronchoalveolar lavage fluid, aiming at deciphering macrophage plasticity in the lung microenvironment, revealed that macrophages with reduced expression of the classical surface markers M1 and M2 exhibit pro-inflammatory gene signatures, and overexpress 15 mitochondrial genes including $m t-C O 3, m t-C O 1, m t-R N R 2$,

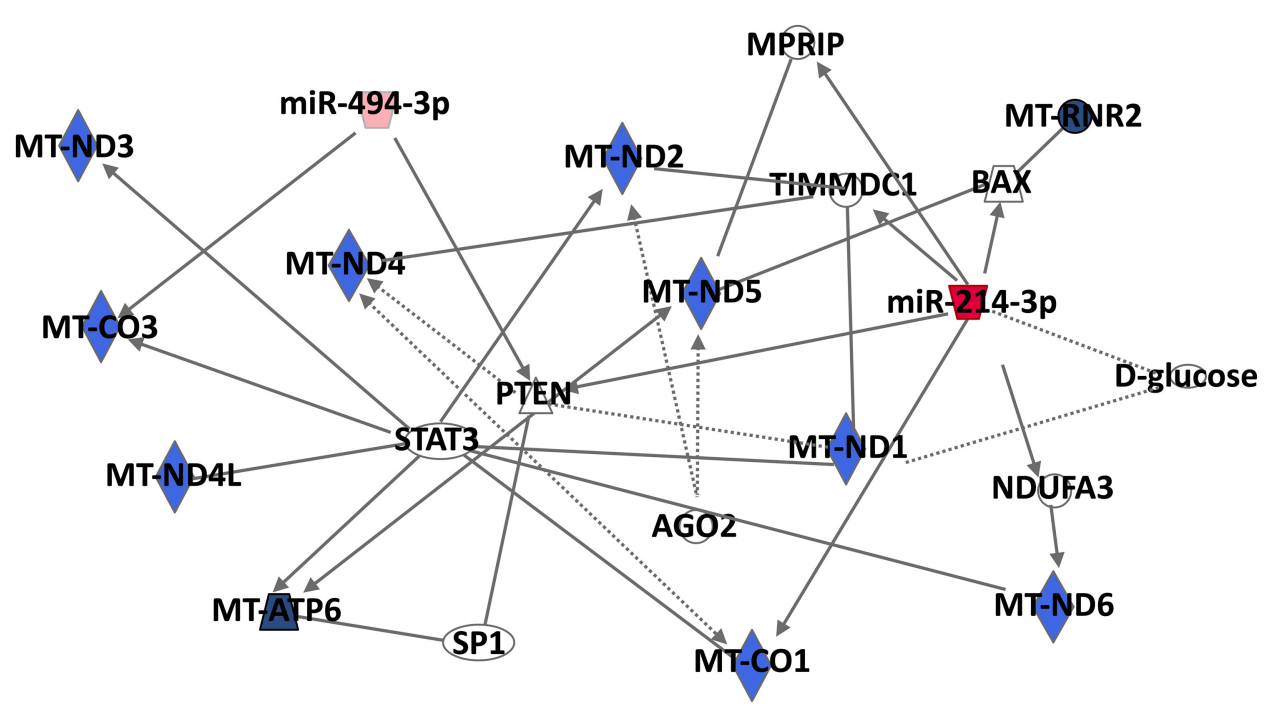

FIGURE 3 | Mt-mRNA-miRNA networks generated with Ingenuity Pathway Analysis (IPA) for the two most described MitomiRs, miR-494, and miR-214. IPA analysis was performed to investigate genes associated with Mt-mRNAs. Filters were set to include either putative, experimentally observed or high-confidence-predicted miRNA-mRNA interaction partners. MiRNAs were colored in red depending on whether they were upregulated in mitochondria fractions following macrophage differentiation. The red color intensity corresponds to the fold change in miRNA expression in the mitochondria-enriched fraction of PMA-differentiated THP-1 macrophages compared to untreated THP-. 
$m t$-ATP6, $m t$-ND1, $m t-N D 2$, and $m t-N D 4 L$, which may contribute to the pathogenesis and manifestations of inflammatory lung diseases such as chronic obstructive pulmonary disease (Takiguchi et al., 2021).

\section{MitomiR TRANSFER TO MACROPHAGE, A NOVEL MODULATORY STRATEGY?}

The clinical significance of this phenomenon was first assessed in a model of Lipopolysaccharide (LPS)-induced lung injury in which the intra-tracheal administration of mesenchymal stem/stromal cells (MSCs) to LPS treated mice was associated with the transfer of mitochondria to alveolar epithelium. MSCs triggered an increase in the concentration of ATP, metabolic activity, also an improvement in lung damage while reducing mortality in the diseased animals. Jackson et al. (2016) observed that mitochondria transferred from MSCs were able to increase the phagocytic capabilities of macrophages, increasing basal respiration and ATP turnover in vitro and in vivo in a murine model of acute respiratory distress syndrome (ARDS). The mechanism by which MSCs transferred mitochondria in this case was independent of CNX43, but related to the establishment of cellular highways (also known as tunneling nanotubules) between both cell types (Jackson et al., 2016). Interestingly, the artificial transfer of mitochondria from MSCs into macrophages, conveys the same metabolic and phagocytic improvement, evidencing a role for mitochondria in macrophage function (Liu D. et al., 2021). Recently, Yuan et al. (2021) showed in a diabetic nephropathy mice model, that mitochondrial transfer from MSCs to macrophages restricts inflammation and alleviates kidney injury via PGC-1 $\alpha$ activation. These results represent strong evidence in favor of the hypothesis that the transfer of mitochondria from MSCs to immune active cells could play a role in the control of immune function mediated by MSCs (Court et al., 2020). However, mitochondrial transfer to macrophages is not specific for MSCs, Brestoff et al. (2021) demonstrated that adipocytes transfer their mitochondria to macrophages in vivo, mediated by heparan sulfates, which regulates white adipose tissue homeostasis, and plays a key role in obesity. In addition, recent studies showed that mitochondria from extracellular vesicles are involved in intercellular communication and immune regulation (She et al., 2021).

Also, mitochondria delivery can be applied following an artificial procedure, known mitoception (Caicedo et al., 2015), where extracted mitochondria from donor cells are transferred into recipient cells. Following this method, freshly isolatedmitochondria from MSCs could be transferred into immune cells including monocytes based on centrifugation and endocytosis. The "mitocepted" cells were able to increase their ATP production while shifting toward a glycolytic pathway. Thus, the

\section{REFERENCES}

Ahn, J. Y., Datta, S., Bandeira, E., Cano, M., Mallick, E., Rai, U., et al. (2021). Release of extracellular vesicle miR-494-3p by ARPE-19 cells with impaired process of mitoception allows a distinct analysis of the functional role of mitochondria, independently from the paracrine and cell-mediated effects from donor cells.

Mitochondrial transfer, whether mediated by cell-to-cell contact or through mitoception, presents a novel modulatory strategy through the delivery of mitomiR(s) to target cells, including macrophages (Jorgensen and Khoury, 2021).

\section{HYPOTHESIS AND CONCLUSION}

Our in silico analysis identified a specific MitomiR signature associated with macrophage functions including differentiation, polarization, recruitment and activation of the inflammatory response. This MitomiR profiling correlated with gene targets within the mitochondrial genome or mitochondrial pathways. Our emitted hypothesis and data suggest a previously unrecognized link between MitomiR and macrophage functions and fate leading to a modulation of the downstream immune responses.

\section{DATA AVAILABILITY STATEMENT}

The datasets presented in this study can be found in online repositories. The names of the repository/repositories and accession number(s) can be found below: NCBI GEO GSE182255.

\section{AUTHOR CONTRIBUTIONS}

ID-R, FA, and MK drafted the manuscript. ID-R performed in silico analyses. All authors were involved in reading and editing the manuscript and approved the final version.

\section{FUNDING}

This work was supported by grants from the Chilean National Agency for Investigation and Development: ANID (Agencia Nacional de Investigación y Desarrollo) (FONDECYT regular, 2021, \#1211749). In addition to research funds from the INSERM (Institut National de la Santé et Recherche Médicale); the University of Montpellier; and the International research project INSERM France-Chile 2020.

\section{ACKNOWLEDGMENTS}

We thank Francesca Velarde from the University of Los Andes, Chile for the critical proofreading of the manuscript.

mitochondria. Biochim. Biophys. Acta Gen. Subj. 1865:129598. doi: 10.1016/j. bbagen.2020.129598

Andrienko, T., Kuznetsov, A. V., Kaambre, T., Usson, Y., Orosco, A., Appaix, F., et al. (2003). Metabolic consequences of functional complexes of mitochondria, 
myofibrils and sarcoplasmic reticulum in muscle cells. J. Exp. Biol. 206, 20592072. doi: 10.1242/JEB.00242

Arsenijevic, D., Onuma, H., Pecqueur, C., Raimbault, S., Manning, B. S., Miroux, B., et al. (2000). Disruption of the uncoupling protein-2 gene in mice reveals a role in immunity and reactive oxygen species production. Nat. Genet. 26, 435-439. doi: 10.1038/82565

Aschrafi, A., Schwechter, A. D., Mameza, M. G., Natera-Naranjo, O., Gioio, A. E., and Kaplan, B. B. (2008). MicroRNA-338 regulates local Cytochrome c Oxidase IV mRNA levels and Oxidative Phosphorylation in the axons of sympathetic neurons. J. Neurosci. 28, 12581-12590. doi: 10.1523/JNEUROSCI.3338-08.2008

Bandiera, S., Rüberg, S., Girard, M., Cagnard, N., Hanein, S., Chrétien, D., et al. (2011). Nuclear outsourcing of RNA interference components to human mitochondria. PLoS One 6:e20746. doi: 10.1371/journal.pone.0020746

Bartel, D. P. (2004). MicroRNAs: genomics, biogenesis, mechanism, and function. Cell 116, 281-297. doi: 10.1016/S0092-8674(04)00045-5

Brestoff, J. R., Wilen, C. B., Moley, J. R., Li, Y., Zou, W., Malvin, N. P., et al. (2021). Intercellular mitochondria transfer to macrophages regulates white adipose tissue homeostasis and is impaired in obesity. Cell Metab. 33, 270-282.e8. doi: 10.1016/j.cmet.2020.11.008

Caicedo, A., Aponte, P. M., Cabrera, F., Hidalgo, C., and Khoury, M. (2017). Artificial mitochondria transfer: current challenges, advances, and future applications. Stem Cells Int. 2017:7610414. doi: 10.1155/2017/7610414

Caicedo, A., Fritz, V., Brondello, J.-M., Ayala, M., Dennemont, I., Abdellaoui, N., et al. (2015). MitoCeption as a new tool to assess the effects of mesenchymal stem/stromal cell mitochondria on cancer cell metabolism and function. Sci. Rep. 5:9073. doi: 10.1038/srep09073

Court, A. C., Le-Gatt, A., Luz-Crawford, P., Parra, E., Aliaga-Tobar, V., Bátiz, L. F., et al. (2020). Mitochondrial transfer from MSCs to T cells induces Treg differentiation and restricts inflammatory response. EMBO Rep. 21:e48052. doi: 10.15252/embr.201948052

Das, S., Bedja, D., Campbell, N., Dunkerly, B., Chenna, V., Maitra, A., et al. (2014). miR-181c regulates the mitochondrial genome, bioenergetics, and propensity for heart failure in vivo. PLoS One 9:e96820. doi: 10.1371/journal.pone.0096820

Das, S., Kohr, M., Dunkerly-Eyring, B., Lee, D. I, Bedja, D., Kent, O. A., et al. (2017). Divergent effects of miR-181 family members on myocardial function through protective cytosolic and detrimental mitochondrial microRNA targets. J. Am. Heart Assoc. 6:e004694. doi: 10.1161/JAHA.116.004694

Dasgupta, N., Peng, Y., Tan, Z., Ciraolo, G., Wang, D., and Li, R. (2015). miRNAs in mtDNA-less cell mitochondria. Cell Death Discov. 1:15004. doi: 10.1038/ cddiscovery.2015.4

Dhir, A., Dhir, S., Borowski, L. S., Jimenez, L., Teitell, M., Rötig, A., et al. (2018). Mitochondrial double-stranded RNA triggers antiviral signalling in humans. Nature 560, 238-242. doi: 10.1038/s41586-018-0363-0

Duroux-Richard, I., Roubert, C., Ammari, M., Présumey, J., Grün, J. R., Häupl, T., et al. (2016). miR-125b controls monocyte adaptation to inflammation through mitochondrial metabolism and dynamics. Blood 128, 3125-3136. doi: 10.1182/ blood-2016-02-697003

Emre, Y., and Nübel, T. (2010). Uncoupling protein UCP2: when mitochondrial activity meets immunity. FEBS Lett. 584, 1437-1442. doi: 10.1016/J.FEBSLET. 2010.03.014

Fang, Y., Qiu, J., Jiang, Z., Xu, S., Zhou, Z., and He, R. (2018). Increased serum levels of miR-214 in patients with PCa with bone metastasis may serve as a potential biomarker by targeting PTEN. Oncol. Lett. 17, 398-405. doi: 10.3892/ ol.2018.9522

Feng, J., Li, L., Ou, Z., Li, Q., Gong, B., Zhao, Z., et al. (2017). IL-25 stimulates M2 macrophage polarization and thereby promotes mitochondrial respiratory capacity and lipolysis in adipose tissues against obesity. Cell. Mol. Immunol. 15, 493-505. doi: 10.1038/cmi.2016.71

Gao, C., Guo, H., Mi, Z., Grusby, M. J., and Kuo, P. C. (2007). Osteopontin induces ubiquitin-dependent degradation of STAT1 in RAW264.7 murine macrophages. J. Immunol. 178, 1870-1881. doi: 10.4049/JIMMUNOL.178.3. 1870

Gao, C., Guo, H., Wei, J., and Kuo, P. C. (2003). Osteopontin inhibits expression of cytochrome c oxidase in RAW 264.7 murine macrophages. Biochem. Biophys. Res. Commun. 309, 120-125. doi: 10.1016/S0006-291X(03)01534-1

Gao, P., Yan, Z., and Zhu, Z. (2020). Mitochondria-associated endoplasmic reticulum membranes in cardiovascular diseases. Front. Cell Dev. Biol. 8:604240. doi: 10.3389/FCELL.2020.604240
Gao, Y., Fang, P., Li, W.-J., Zhang, J., Wang, G.-P., Jiang, D.-F., et al. (2020). LncRNA NEAT1 sponges miR-214 to regulate M2 macrophage polarization by regulation of B7-H3 in multiple myeloma. Mol. Immunol. 117, 20-28. doi: 10.1016/j.molimm.2019.10.026

Geiger, J., and Dalgaard, L. T. (2017). Interplay of mitochondrial metabolism and microRNAs. Cell. Mol. Life Sci. 74, 631-646. doi: 10.1007/s00018-016-2342-7

Gray, M. W., Burger, G., and Lang, B. F. (1999). Mitochondrial evolution. Science 283, 1476-1481. doi: 10.1126/SCIENCE.283.5407.1476

Gustafsson, ÅB., and Gottlieb, R. A. (2008). Heart mitochondria: gates of life and death. Cardiovasc. Res. 77, 334-343. doi: 10.1093/CVR/CVM005

He, W., Heinz, A., Jahn, D., and Hiller, K. (2021). Complexity of macrophage metabolism in infection. Curr. Opin. Biotechnol. 68, 231-239. doi: 10.1016/J. COPBIO.2021.01.020

Jackson, M. V., Morrison, T. J., Doherty, D. F., McAuley, D. F., Matthay, M. A., Kissenpfennig, A., et al. (2016). Mitochondrial transfer via tunneling nanotubes is an important mechanism by which mesenchymal stem cells enhance macrophage phagocytosis in the in vitro and in vivo models of ARDS. Stem Cells 34, 2210-2223. doi: 10.1002/stem.2372

Jha, A. K., Huang, S. C. C., Sergushichev, A., Lampropoulou, V., Ivanova, Y., Loginicheva, E., et al. (2015). Network integration of parallel metabolic and transcriptional data reveals metabolic modules that regulate macrophage polarization. Immunity 42, 419-430. doi: 10.1016/J.IMMUNI.2015.02.005

Jorgensen, C., and Khoury, M. (2021). Musculoskeletal Progenitor/Stromal Cell-Derived Mitochondria Modulate Cell Differentiation and Therapeutical Function. Front. Immunol. 12:606781. doi: 10.3389/FIMMU.2021.606781

Kadenbach, B., and Hüttemann, M. (2015). The subunit composition and function of mammalian cytochrome c oxidase. Mitochondrion 24, 64-76. doi: 10.1016/j. mito.2015.07.002

Kelley, N., Jeltema, D., Duan, Y., and He, Y. (2019). The NLRP3 Inflammasome: an overview of mechanisms of activation and regulation. Int. J. Mol. Sci. 20:3328. doi: 10.3390/IJMS20133328

Lee, H., Tak, H., Park, S. J., Jo, Y. K., Cho, D. H., and Lee, E. K. (2017). microRNA200a-3p enhances mitochondrial elongation by targeting mitochondrial fission factor. BMB Rep. 50, 214-219. doi: 10.5483/BMBRep.2017.50.4.006

Li, J., Donath, S., Li, Y., Qin, D., Prabhakar, B. S., and Li, P. (2010). miR-30 Regulates Mitochondrial Fission through Targeting p53 and the DynaminRelated Protein-1 Pathway. PLoS Genet. 6:e1000795. doi: 10.1371/journal.pgen. 1000795

Li, J., Li, Y., Jiao, J., Wang, J., Li, Y., Qin, D., et al. (2014). Mitofusin 1 is negatively regulated by microrna 140 in Cardiomyocyte Apoptosis. Mol. Cell. Biol. 34, 1788-1799. doi: 10.1128/MCB.00774-13

Liu, D., Gao, Y., Liu, J., Huang, Y., Yin, J., Feng, Y., et al. (2021). Intercellular mitochondrial transfer as a means of tissue revitalization. Signal Transduct. Target. Ther. 6:65. doi: 10.1038/s41392-020-00440-Z

Liu, Y., Xu, R., Gu, H., Zhang, E., Qu, J., Cao, W., et al. (2021). Metabolic reprogramming in macrophage responses. Biomark. Res. 9:1. doi: 10.1186/ S40364-020-00251-Y

Lu, S., Gao, Y., Huang, X., and Wang, X. (2014). Cantharidin exerts AntiHepatocellular Carcinoma by Mir-214 modulating macrophage polarization. Int. J. Biol. Sci. 10, 415-425. doi: 10.7150/ijbs.8002

Maniataki, E. (2005). Human mitochondrial tRNAMet is exported to the cytoplasm and associates with the Argonaute 2 protein. RNA 11, 849-852. doi: 10.1261/ rna. 2210805

Mehla, K., and Singh, P. K. (2019). Metabolic Regulation of Macrophage Polarization in Cancer. Trends Cancer 5, 822-834. doi: 10.1016/J.TRECAN. 2019.10.007

Meng, L., Lu, C., Wu, B., Lan, C., Mo, L., Chen, C., et al. (2021). Taurine Antagonizes Macrophages M1 Polarization by Mitophagy-Glycolysis Switch Blockage via Dragging SAM-PP2Ac Transmethylation. Front. Immunol. 12:648913. doi: 10.3389/FIMMU.2021.648913

Mukherjee, S., Basu, S., Home, P., Dhar, G., and Adhya, S. (2007). Necessary and sufficient factors for the import of transfer RNA into the kinetoplast mitochondrion. EMBO Rep. 8, 589-595. doi: 10.1038/sj.embor.7400979

Pecqueur, C., Alves-Guerra, C., Ricquier, D., and Bouillaud, F. (2009). UCP2, a metabolic sensor coupling glucose oxidation to mitochondrial metabolism? IUBMB Life 61, 762-767. doi: 10.1002/IUB.188

Purohit, P. K., Edwards, R., Tokatlidis, K., and Saini, N. (2019). MiR-195 regulates mitochondrial function by targeting mitofusin-2 in breast 
cancer cells. RNA Biol. 16, 918-929. doi: 10.1080/15476286.2019.160 0999

Qing, J., Zhang, Z., Novák, P., Zhao, G., and Yin, K. (2020). Mitochondrial metabolism in regulating macrophage polarization: an emerging regulator of metabolic inflammatory diseases. Acta Biochim. Biophys. Sin. (Shanghai) 52, 917-926. doi: 10.1093/abbs/gmaa081

Ramond, E., Jamet, A., Coureuil, M., and Charbit, A. (2019). Pivotal role of mitochondria in macrophage response to bacterial pathogens. Front. Immunol. 10:2461. doi: 10.3389/fimmu.2019.02461

Roger, A. J., Muñoz-Gómez, S. A., and Kamikawa, R. (2017). The origin and diversification of mitochondria. Curr. Biol. 27, R1177-R1192. doi: 10.1016/j. cub.2017.09.015

Self-Fordham, J. B., Naqvi, A. R., Uttamani, J. R., Kulkarni, V., and Nares, S. (2017). MicroRNA: dynamic regulators of macrophage polarization and plasticity. Front. Immunol. 8:1062. doi: 10.3389/fimmu.2017.01062

Shao, G., Zhou, C., Ma, K., Zhao, W., Xiong, Q., Yang, L., et al. (2020). MiRNA494 enhances M1 macrophage polarization via Nrdp1 in ICH mice model. J. Inflamm. 17:17. doi: 10.1186/s12950-020-00247-3

She, Z., Xie, M., Hun, M., Abdirahman, A. S., Li, C., Wu, F., et al. (2021). Immunoregulatory effects of mitochondria transferred by extracellular vesicles. Front. Immunol. 11:628576. doi: 10.3389/fimmu.2020.628576

Shepherd, D. L., Hathaway, Q. A., Pinti, M. V., Nichols, C. E., Durr, A. J., Sreekumar, S., et al. (2017). Exploring the mitochondrial microRNA import pathway through Polynucleotide Phosphorylase (PNPase). J. Mol. Cell. Cardiol. 110, 15-25. doi: 10.1016/j.yjmcc.2017.06.012

Sheridan, C., and Martin, S. J. (2010). Mitochondrial fission/fusion dynamics and apoptosis. Mitochondrion 10, 640-648. doi: 10.1016/j.mito.2010.08.005

Subramanian, N., Natarajan, K., Clatworthy, M. R., Wang, Z., and Germain, R. N. (2013). The Adaptor MAVS Promotes NLRP3 mitochondrial localization and inflammasome activation. Cell 153, 348-361. doi: 10.1016/J.CELL.2013.02.054

Takiguchi, H., Yang, C. X., Yang, C. W. T., Sahin, B., Whalen, B. A., Milne, S., et al. (2021). Macrophages with reduced expressions of classical M1 and M2 surface markers in human bronchoalveolar lavage fluid exhibit pro-inflammatory gene signatures. Sci. Rep. 11:8282. doi: 10.1038/s41598-021-87720-y

Van den Bossche, J., Baardman, J., Otto, N. A., van der Velden, S., Neele, A. E., van den Berg, S. M., et al. (2016). Mitochondrial dysfunction prevents repolarization of inflammatory macrophages. Cell Rep. 17, 684-696. doi: 10.1016/J.CELREP. 2016.09.008

Veremeyko, T., Siddiqui, S., Sotnikov, I., Yung, A., and Ponomarev, E. D. (2013). IL-4/IL-13-Dependent and Independent Expression of miR-124 and Its Contribution to M2 Phenotype of Monocytic Cells in Normal Conditions and during Allergic Inflammation. PLoS One 8:e81774. doi: 10.1371/journal.pone. 0081774

Wan, Q., Xu, T., Ding, W., Zhang, X., Ji, X., Yu, T., et al. (2019). miR-499-5p Attenuates Mitochondrial Fission and Cell Apoptosis via p21 in Doxorubicin Cardiotoxicity. Front. Genet. 9:734. doi: 10.3389/fgene.2018.00734

Wang, W.-X., Prajapati, P., Nelson, P. T., and Springer, J. E. (2020). The mitochondria-associated ER membranes are novel subcellular locations enriched for inflammatory-responsive MicroRNAs. Mol. Neurobiol. 57, 29963013. doi: 10.1007/s12035-020-01937-y

Wei, J., Guo, H., and Kuo, P. C. (2002). Endotoxin-Stimulated Nitric Oxide Production Inhibits Expression of Cytochrome c Oxidase in ANA-1 Murine Macrophages. J. Immunol. 168, 4721-4727. doi: 10.4049/jimmunol.168.9.4721

Wei, Q., Sun, H., Song, S., Liu, Y., Liu, P., Livingston, M. J., et al. (2018). MicroRNA-668 represses MTP18 to preserve mitochondrial dynamics in ischemic acute kidney injury. J. Clin. Invest. 128, 5448-5464. doi: 10.1172/ JCI121859

Yamamoto, H., Morino, K., Nishio, Y., Ugi, S., Yoshizaki, T., Kashiwagi, A., et al. (2012). MicroRNA-494 regulates mitochondrial biogenesis in skeletal muscle through mitochondrial transcription factor A and Forkhead box j3. Am. J. Physiol. Metab. 303, E1419-E1427. doi: 10.1152/ajpendo.00097.2012

Yang, X., Cai, S., Shu, Y., Deng, X., Zhang, Y., He, N., et al. (2021). Exosomal miR487 a derived from $\mathrm{m} 2$ macrophage promotes the progression of gastric cancer. Cell Cycle 20, 434-444. doi: 10.1080/15384101.2021.1878326

Yuan, Y., Yuan, L., Li, L., Liu, F., Liu, J., Chen, Y., et al. (2021). Mitochondrial transfer from mesenchymal stem cells to macrophages restricts inflammation and alleviates kidney injury in diabetic nephropathy mice via PGC-1 $\alpha$ activation. Stem Cells 39, 913-928. doi: 10.1002/stem.3375

Zhang, S., He, K., Zhou, W., Cao, J., and Jin, Z. (2019). miR-494-3p regulates lipopolysaccharide-induced inflammatory responses in RAW264.7 cells by targeting PTEN. Mol. Med. Rep. 19, 4288-4296. doi: 10.3892/mmr.2019. 10083

Zhao, Y., Ponnusamy, M., Liu, C., Tian, J., Dong, Y., Gao, J., et al. (2017). MiR-485$5 \mathrm{p}$ modulates mitochondrial fission through targeting mitochondrial anchored protein ligase in cardiac hypertrophy. Biochim. Biophys. Acta Mol. Basis Dis. 1863, 2871-2881. doi: 10.1016/j.bbadis.2017.07.034

Conflict of Interest: MK was the chief scientific officer of Cells for Cells and Regenero, the Chilean Consortium for Regenerative Medicine.

The remaining authors declare that the research was conducted in the absence of any commercial or financial relationships that could be construed as a potential conflict of interest.

Publisher's Note: All claims expressed in this article are solely those of the authors and do not necessarily represent those of their affiliated organizations, or those of the publisher, the editors and the reviewers. Any product that may be evaluated in this article, or claim that may be made by its manufacturer, is not guaranteed or endorsed by the publisher.

Copyright (c) 2021 Duroux-Richard, Apparailly and Khoury. This is an open-access article distributed under the terms of the Creative Commons Attribution License (CC BY). The use, distribution or reproduction in other forums is permitted, provided the original author(s) and the copyright owner(s) are credited and that the original publication in this journal is cited, in accordance with accepted academic practice. No use, distribution or reproduction is permitted which does not comply with these terms. 